\title{
Mariner 1969 Infrared Radiometer Results : Temperatures and Thermal Properties of the Martian Surface
}

\author{
G. Neugebauer and G. MÜNCH \\ California Institute of Technology, Pasadena, California \\ H. KIEFFER \\ University of California, Los Angeles, California \\ S. C. Chase, Jr. \\ Santa Barbara Research Center, Goleta, California \\ AND \\ E. MINER \\ Jet Propulsion Laboratory, Pasadena, California
}

(Received 18 May 1971)

\begin{abstract}
The reduced data of the Mariner 6 and 7 Infrared Radiometer Experiments are presented, along with a discussion of the reduction and calibration procedures. Evidence is presented showing that the surface of Mars is strongly nonhomogeneous in its thermal properties, on scales ranging from those of the classical light and dark areas to the limit of resolution of the radiometers. On the sunlit side, the mean thermal inertia, for admissible bolometric albedos, is $0.006\left(\mathrm{cal} \mathrm{cm}^{-2} \mathrm{sec}^{-1}{ }^{\circ} \mathrm{K}^{-1}\right)$. The dark areas Syrtis Major and Mare Tyrrhenum, observed at night, require thermal inertias as high as 0.010 . The temperatures measured over the circular basin Hellas require a bolometric albedo of 0.40 and also a high thermal inertia. The temperature measured over the south polar cap, $148^{\circ} \mathrm{K}$, provides evidence that the major constituent of the frost deposit is $\mathrm{CO}_{2}$.
\end{abstract}

$T$ $\mathrm{HE}$ infrared radiometers carried on the Mariner 6 and 7 spacecraft were designed to measure the energy emitted by Mars in two broad wavelength bands of its thermal spectrum. Preliminary presentations of the results obtained have been published (Neugebauer et al. 1969a,b); in this paper, refined estimates of the temperatures characterizing the top layer of the Martian soil are presented. On the basis of these final results the thermophysical properties of the various Martian terrains are inferred and discussed in relation to their visual characteristics. Special consideration is given to the measurements obtained over the south polar cap flyby of Mariner 7 to derive the temperature of the frost deposit forming the cap.

\section{INSTRUMENTATION}

A complete description of the infrared radiometer flown on both Mariners 6 and 7 has been given by Chase (1969) and only those characteristics which are essential for an understanding of the data will be discussed here. The radiometers each consisted of two refracting 1-inch aperture telescopes, one of which accepted radiation in the wavelength range 8.1 to $12.5 \mu$ and the other in the range 17.9 to $25.1 \mu$. Interference filters were used to isolate these wavelength intervals which were chosen to avoid atmospheric $\mathrm{CO}_{2}$ emission. The optical axes of the telescopes were parallel to each other and were mounted on the scan platforms of the spacecraft in such a way as to be parallel to the optical axes of the TV imaging cameras. The fields of view of the telescopes, defined by the dimensions of the thermopile detectors in conjunction with the entrance optics, subtended angles of approximately $14 \mathrm{mrad}$ full-width at half peak response.

The radiometric measurements were made through a rotatable mirror, such that (i) the planet, (ii) the space at right angles to the planet direction, or (iii) a serrated thermal calibration plate could be observed. A sequence of measurements started with an observation of space (ii) for $4.2 \mathrm{sec}$. The signal obtained during this time was used to establish an electrical zero reference and all other subsequent telemetered data were differences from this "space" value. After the space observation, the mirror was rotated $90^{\circ}$ to view the planet, where 13 measurements separated by 2.1 sec were made in each wavelength range. The mirror was then rotated another $90^{\circ}$ to measure the energy emitted by the serrated calibration plate. Fourteen further measurements of the planet then followed, after which space was again viewed and a new cycle initiated. The entire sequence lasted $63 \mathrm{sec}$, and once during such a sequence, while space was being viewed, the temperature of the calibration plate, as measured by a thermistor imbedded in the plate, was recorded.

Before the spacecraft were launched, the radiometers were calibrated by measuring the response to radiation from conical blackbodies whose temperatures were varied from 100 to $330^{\circ} \mathrm{K}$; the gains of the amplifiers were adjusted to accommodate signals over this temperature range. As a result of these tests it was established that the responses of the radiometer systems were linear within $1 \%$ with the energy received. The gains of the systems, which were compensated for temperature variations, were found to vary smoothly and by less than $7 \%$ for instrument temperature between -30 and $+30^{\circ} \mathrm{C}$. 


\section{REDUCTION TO BRIGHTNESS TEMPERATURES}

The radiometers on both spacecraft worked essentially as planned; the system gains derived from the signals obtained by the radiometers while viewing the calibration plate were within $10 \%$ of those obtained from the ground-based calibrations. Furthermore, the dependence of the gains on the instrument temperatures, as determined from a wide range of pre-encounter flight data, agreed with the dependence as measured before the flights. The thermistor-derived temperatures for the reference plate were corroborated by engineering temperature sensors located on the spacecraft near the instrument.

Preflight laboratory measurements, as well as the actual transitions onto the planet,' showed that the radiometers had a finite response outside the nominal $14 \mathrm{mrad}$ field of view; if a uniform source of infinite extent were viewed, the response beyond $1^{\circ}$ from the center of the field of view could contribute up to $30 \%$ of the signal. The response of the 18-25- $\mu$ channels extended to larger distances than those of the 8-14- $\mu$ channels. The angular dependence of the response was actually mapped in the laboratory only to a distance of about $20 \mathrm{mrad}$ from the optical axes. The response at larger distances from the center of the field of view was known to be significant, but could not be mapped in detail before the flight because of technical difficulties. Thus the total response of each radiometer was determined by combining laboratory measurements of the near response with the far response derived from signals observed while crossing onto the planet. This response, tabulated at a grid of 3846 points, was then used in an iterative correction procedure to account for the influence of the radiation observed outside the nominal field. The radiation of the planet was approximated by a model, described in Sec. IIIB, which represents the average thermal properties of the planet and this model was then used to calculate the contribution by radiation outside the nominal central field of view to the observed energy. The correction was of little importance except near the limbs of the planet and during passage over the edge of the polar cap. It is considered unreliable whenever it exceeds $15 \%$ of the observed signal and the corresponding temperatures have been arbitrarily disregarded except for those obtained during the transition onto the polar cap. These data will be discussed separately below.

The scanning patterns on Mars of both Mariners 6 and 7 were broken up into five swaths during each of which the pointing angles of the scan platform relative to the spacecraft were held fixed. During the slues between these swaths there was some evidence of electrical interference with the Mariner 7 radiometer data and as a result the data obtained during the actual slues of both spacecraft have been omitted as were the data cycles initiated during the slues.
For all of the data, the detector noise was much less than the uncertainty introduced by the 10-bit digitization of the signal. Over the bulk of the observations, when temperatures exceeded $200^{\circ} \mathrm{K}$, this uncertainty amounts to approximately $0.3^{\circ} \mathrm{K}$; thus, during one swath, $0.3^{\circ} \mathrm{K}$ is probably a good estimate of the relative accuracy of the temperatures observed in one wavelength interval. The systematic errors, which result in an uncertainty in the absolute value of the temperatures, are more difficult to estimate. If, for example, the preflight laboratory calibrations were assumed to hold during the encounter, the derived temperatures could be altered by as much as $5^{\circ} \mathrm{K}$. This number is a likely estimate of the uncertainty in the absolute brightness temperatures except during observations of the polar cap. The signals obtained from this area, even in the 18-25- $\mu$ channel, amount to only $5 \%$ of the signal obtained near the equator of the planet and thus are highly susceptible to systematic errors. The problems associated with the polar pass data of Mariner 7 will be discussed in detail separately below (Sec. IIId).

The brightness temperatures finally adopted for Mariners 6 and 7 together with the encounter geometry have been tabulated and are available from the National Space Data Center at Goddard Space Flight Center, Greenbelt, Maryland.

The brightness temperatures obtained by the Mariner radiometers in the 8-12- $\mu$ range are in excellent agreement with those measured from the ground by Sinton and Strong (1960) in the range 7 to $13 \mu$ with respect to laboratory blackbodies. Specifically, the temperature of $294^{\circ} \mathrm{K}$ measured by these authors for Meridiani Sinus at the subsolar point, should be compared with the value $T_{10}=288^{\circ} \mathrm{K}$ obtained with Mariner 7 . Allowing for the slightly larger heliocentric distance at the time of the Mariner flyby (1.43 versus 1.41 A.U.), the difference between the spacecraft and ground-based measurements is only $4^{\circ} \mathrm{K}$, well within the intrinsic calibration errors of the ground-based determination.

The smoothing effect of the extended fields of view also limited the capability of detecting localized thermal inhomogeneities on the Martian surface. In fact, of course, the response to a given local surface-temperature fluctuation depends on the unknown complexion of the surface temperature over a relatively extended area. The smoothing effects can, however, be estimated theoretically by convolving the instrumental angular response with a surface-temperature distribution which contains an arbitrary temperature perturbation. The nominal conductive-radiative model used to determine the effects of the field of view was, therefore, modified by assuming the temperature of various square fields on the planetary surface to be raised by an arbitrary amount $\delta T$. The calculations show that if the nominal field of $14 \mathrm{mrad}$ is filled with such a perturbation, the apparent increase observed to a $270^{\circ} \mathrm{K}$ scene in the 
8-14- $\mu$ channel would be $0.3 \delta T$ while that observed in the 18-25- $\mu$ channel would be only $0.2 \delta T$. The minimum temperature perturbation which is detectable also depends on the number of sample points taken across the perturbation. At a typical slant range of $4000 \mathrm{~km}$, near the middle of the encounter, a $100-\mathrm{km}$ wide (25-mrad) surface feature would have been observed approximately ten times with more than half its peak intensity. Assuming an uncertainty in the temperatures of about $0.3^{\circ} \mathrm{K}$, an examination of the calculations shows that an inhomogeneity of $1^{\circ} \mathrm{K}$ over a $100-\mathrm{km}$ $\times 100-\mathrm{km}$ square would be easily detectable $(\sim 4 \sigma)$.

\section{DISCUSSION}

\section{A. Emissivity and Phase Function}

The thermal emission of the Martian surface is expected to differ from that of a smooth blackbody in the wavelength dependence of its emissivity and in its directional properties. For instance, if the planetary surface is cratered at a sufficiently small scale, the intensity of thermal emission will depend on the angle of emission. Thus, measurements of the thermal lunar emission (Pettit and Nicholson 1930; Sinton 1962) show an emission angle dependence which has been interpreted in terms of the structural properties of the lunar surface (Smith 1967; Buhl, Welch, and Rea 1968).

As a result of the above consideration, the brightness temperatures measured by the radiometers cannot be identified as the kinetic temperatures of the Martian soil. Unfortunately, the measurements obtained do not suffice to give unique and independent estimates of either the emissivity of the surface or of the possible dependence on the angle of the emissivity. Although a major conclusion which will be drawn from the radiometer data is that the Martian surface is strongly nonhomogeneous in its thermal properties, it is meaningful to define the average values of these parameters to serve as a basis for estimating the extent of possible inhomogeneities in the thermal structure of the planet.

Laboratory measurements have established that the emissivity in the $8-13-\mu$ range, $\epsilon_{10}$, of a variety of terrestrial powdered minerals is about 0.90 (Hovis and Callahan 1966). A kinetic temperature $T_{10}$ was, therefore, derived from the 8-12- $\mu$ data by taking $\epsilon_{10}$ to be 0.90 ; the emissivity in the $18-25-\mu$ channel, $\epsilon_{20}$, was then varied to minimize the difference, in a mean sense over all the data, between $T_{20}$, the kinetic temperature derived from the $18-25-\mu$ data, and $T_{10}$.

The difference $\Delta T \equiv T_{20}-T_{10}$ is also sensitive to the phase function, and a dependence of the thermal energy emitted at an angle EM proportional to $\cos ^{Q-1}(\mathrm{EM})$ was included in the minimization of $\Delta T$. A blackbody radiator has $Q=1$; the lunar surface has $Q=1.20 \pm 0.05$ (Pettit and Nicholson 1930). The parameters which gave the lowest mean residual and the least resultant dependence on emission angle for both the Mariner 6 and 7 data, were $\epsilon_{10}=0.90$ (assumed), $\epsilon_{20}=0.88 \pm 0.03, Q=1.10 \pm 0.07$. These parameters have been used to derive the temperatures which will subsequently be discussed and which are plotted in Figs. 1 and 2.

The temperature differences $\Delta T$ were typically in the range $\pm 2^{\circ} \mathrm{K}$, but showed some systematic trends. Generally $\Delta T$ was negative on the sunlit portion of the planet and positive on the dark side; some examples are included in Plates II-IV. It is not clearly established whether this day-night difference is real or whether it is partly instrumental, since thermal offsets produced by the varying planetary irradiation on the spacecraft could have effected a zero shift of a comparable magnitude. The effect was, however, the same for both Mariners 6 and 7.

One area (longitude $34^{\circ} \mathrm{W}$, latitude $-12^{\circ}$ ) was observed by the Mariner 6 radiometer at emission angles of both $30^{\circ}$ and $56^{\circ}$, thus giving an independent check on the phase function. The value of $Q$ derived from the data of this area is $1.17 \pm 0.05$, in satisfactory agreement with the average value obtained above.

\section{B. Thermophysical Properties of the Martian Surface}

In this section, the kinetic temperatures derived above are compared with those of models consisting of a homogeneous conductive solid heated by insolation and radiating to space. Similar calculations for Mars have been made by Sinton and Strong (1960), Leovy (1966), and Morrison et al. (1969) largely using the methods or solutions developed in studies of lunar temperature variations by Wesselink (1948) and Jaeger (1953). A new set of calculations was carried out for this work in order to have a self-consistent set of models at the appropriate season to compare with the Mariner measurements.

The following units are used throughout this work: thermal conductivity $K$, cal cm${ }^{-1} \mathrm{sec}^{-1}{ }^{\circ} \mathrm{K}^{-1}$; specific heat $c$, cal $\mathrm{gm}^{-1}{ }^{\circ} \mathrm{K}^{-1}$; density $\rho, \mathrm{gm} \mathrm{cm}^{-3}$; thermal inertia, $I=(K \rho c)^{\frac{1}{2}}$, $\mathrm{cal} \mathrm{cm}^{-2} \mathrm{sec}^{-\frac{1}{2}}{ }^{\circ} \mathrm{K}^{-1}$.

The only free parameters in the thermal model are the surface emissivity $\epsilon$, the bolometric albedo $A$, and the thermal inertia $I=(K \rho c)^{\frac{1}{2}}$, where $K$ is the thermal conductivity, $\rho$ the density, and $c$ the specific heat.

The dependence of the model temperatures on emissivity is comparatively minor and a value 0.90 was adopted to be consistent with the radiometry data reduction. For temperatures less than $300^{\circ} \mathrm{K}, 90 \%$ of blackbody radiation occurs at wavelengths greater than $7 \mu$. Infrared reflectance measurements of a variety of pulverized rock specimens indicate that the average emissivity from 7 to $22 \mu$ is about equal to or slightly greater than the emissivity across the 8-14- $\mu$ wavelength region (Hovis and Callahan 1966). Varying the emissivity within the range consistent with the observations $(0.88$ to 0.92$)$ has an effect on the model tempera- 


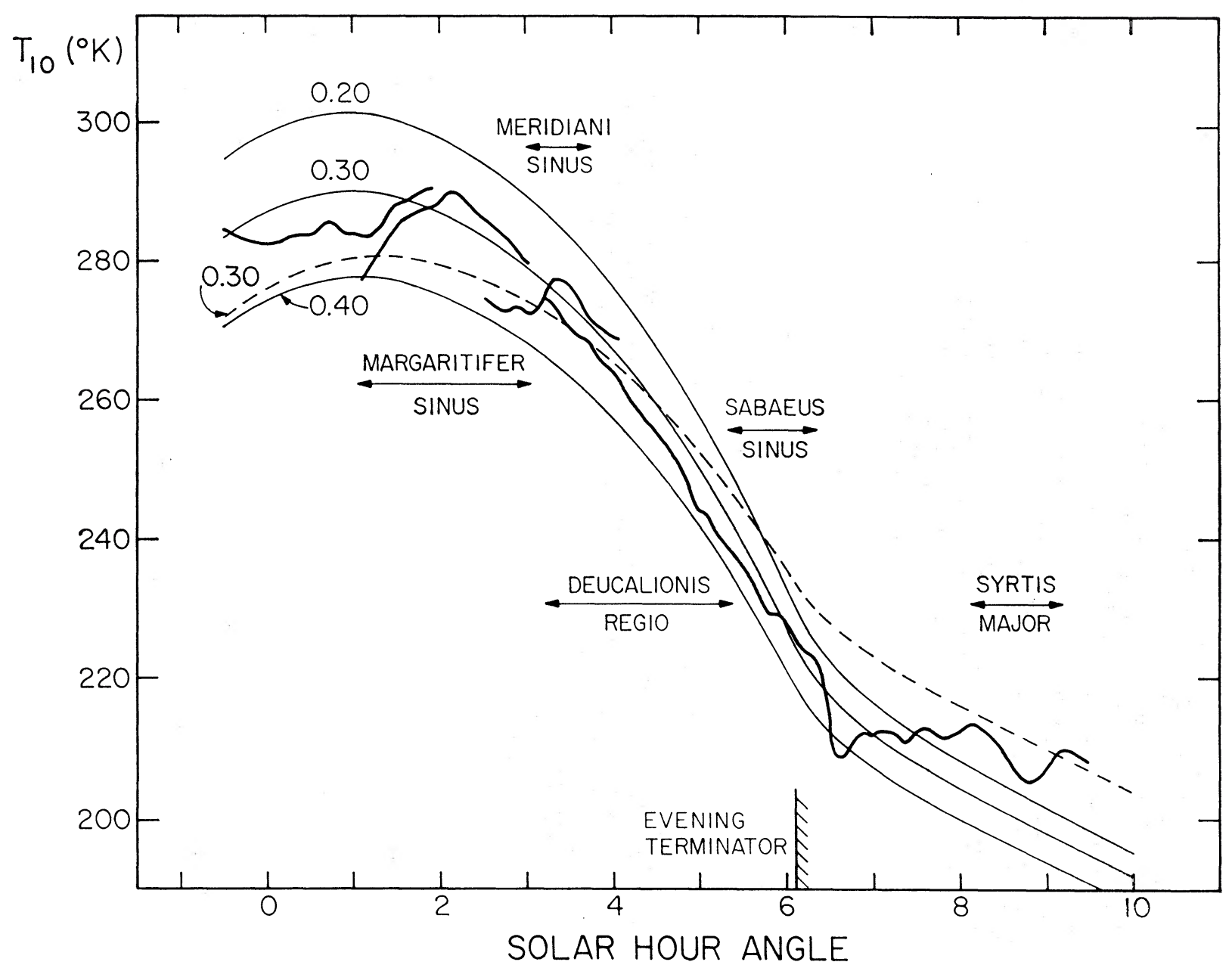

FIG. 1. The kinetic temperatures $T_{10}$ derived from the 8-12- $\mu$ data of Mariner 6 are shown as a function of local time. Model temperatures are shown for values of the thermal inertia of 0.006 (solid lines) and 0.010 (dashed line). Each model curve is identified by the assumed value of the bolometric albedo. The model temperatures have been drawn to be continuous since the differences arising from the different latitudes of individual swaths are too small to be shown graphically. The maximum difference occurs at Margaritifer Sinus where model temperatures are $2^{\circ} \mathrm{K}$ lower than shown.

tures of no more than $3^{\circ}$ and is nearly constant through the day. The effect of atmospheric radiation was included explicitly by a constant atmospheric back radiation equal to 0.01 of the local noon solar flux. The inclusion in the model of the interaction with an atmosphere, of inhomogeneities in depth, and of temperature-dependent physical properties are discussed in the Appendix. The predicted surface temperatures are there shown to depend almost exclusively on the assumed thermal inertia and bolometric albedo; thus models incorporating other independent free variables are not considered here.

The surface temperatures predicted for a family of thermal parameters are shown in Figs. 1 and 2 together with the data obtained from Mariners 6 and 7. Generally, the observations of the daytime temperatures are consistent with models which have a thermal inertia 0.006 and bolometric albedos ranging from 0.2 to 0.4 . The bolometric albedo for the entire planet has been estimated by de Vaucouleurs (1964) from ground-based measurements to be 0.29 . For specific dark and light areas, only measurements of spectral reflectivity are available (McCord and Westphal 1971; Younkin 1970; McCord, Elias, and Westphal 1971); on the unlikely assumption that all areas have the same phase integral, these would be proportional to the corresponding albedos. McCord and Westphal (1971) used their data to estimate monochromatic geometrical albedos for selected areas of Mars. Between 0.7 and $2.5 \mu$ they conclude that for Syrtis Major the geometric albedo is 0.15 , while the light area Arabia is consistent with a geometric albedo of 0.4 . The albedos required by the radiometric data are seen to be consistent with the measured relative brightness of the light and dark surface areas. The temperature peak observed at Meridiani Sinus near noon (Fig. 2) requires an albedo of 0.20 for a model with $I=0.006$. This is the lowest albedo derived from daytime observations and agrees 


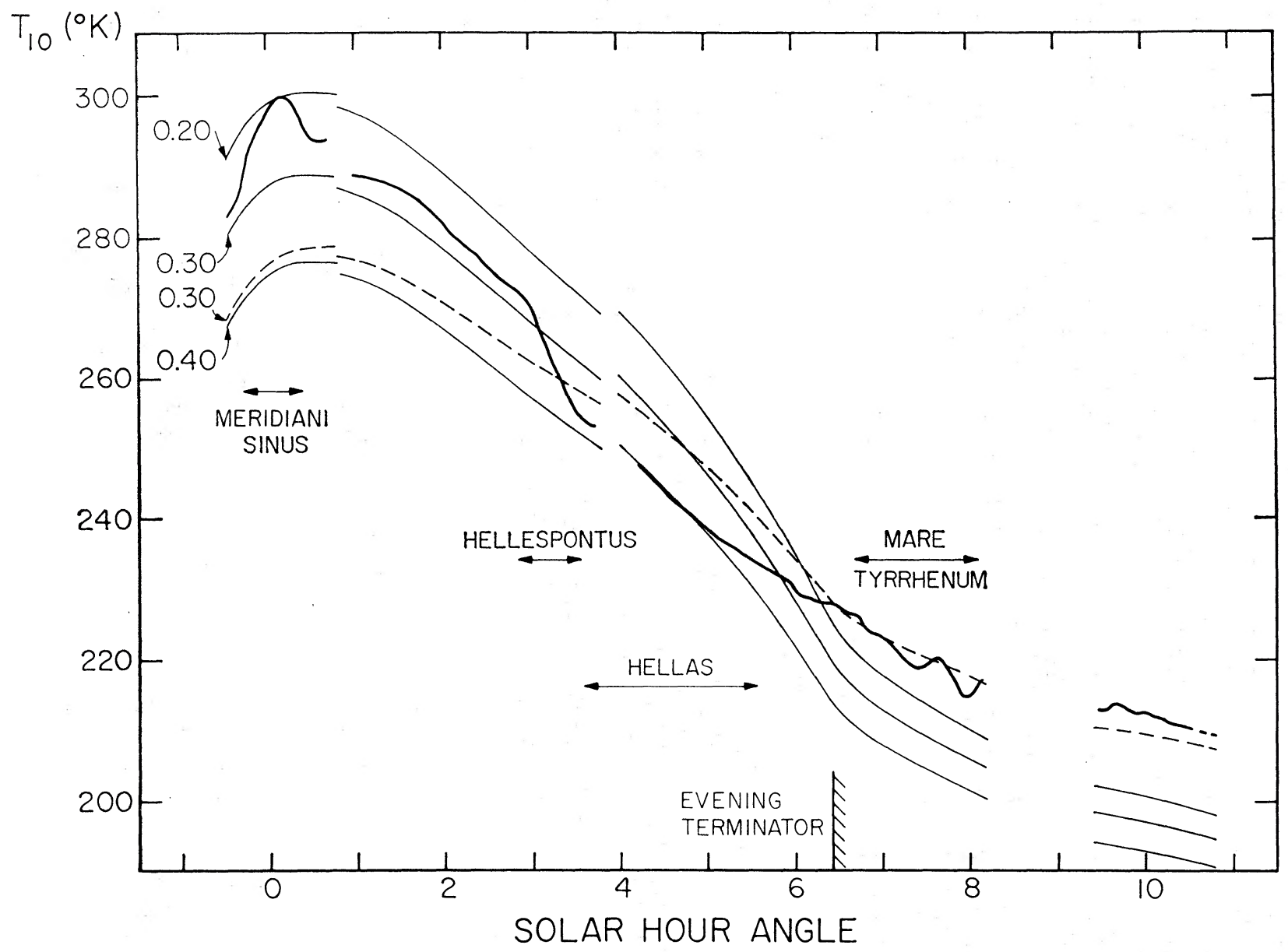

FIg. 2. Same as Fig. 1 but Mariner 7 data.

well with the albedo estimated by extrapolating, in both wavelength and phase angle, terrestrial photometric observations. The particular areas of Deucalionis Regio and Hellas, both classically light, require albedos of 0.34 and 0.40 . These features were observed at local times of approximately $4.5 \mathrm{~h}$ after noon, a time when the model calculations are insensitive to the assumed inertia. Thus, these high albedos must be considered as being well established.

Although the temperature at local times of $4.5 \mathrm{~h}$ is primarily determined by the albedo, the rate of change of temperature with local time is determined almost entirely by the thermal inertia. The Mariner TV pictures (Cutts et al. 1971) show that the visual reflectivity across Hellas is nearly constant. If the same is true for the bolometric albedo, the temperature variations observed between 4.2 and $5.4 \mathrm{~h}$ local time suggest a thermal inertia of about 0.010 , definitely greater than the Martian average.

The observed nighttime temperatures are systematically higher than those predicted by the models which bracket the daytime measurements. The tracks of both spacecraft were selected so the nighttime passage was over the classically dark areas of Syrtis Major (Mariner 6) and Mare Tyrrhenum (Mariner 7). McCord and Westphal (1971) suggest that the geometric albedo of Syrtis Major over the relevant wavelength region of 0.7 to $2.5 \mu$ is 0.15 . If the bolometric albedo has this value, an inertia of 0.009 is inferred for Syrtis Major from the observation at $3 \mathrm{~h}$ past the evening terminator when temperatures are relatively insensitive to albedo. The albedo for Mare Tyrrhenum is not as well established as that for Syrtis Major but may be slightly higher (McCord and Westphal 1971). An examination of Fig. 2 shows that this would imply an inertia even higher than 0.010. Morrison et al. (1969) have suggested that the dark Martian areas have higher thermal inertias than average light areas.

In this discussion, only the ideal case of a surface composed of vertically homogeneous material with constant thermal inertia and diffusivity has been considered; each scene viewed by the radiometer was assumed to be laterally homogeneous. Mars cannot be expected to conform to this ideal case described by a minimum number of parameters. Relaxing each of these assumptions is qualitatively equivalent to mixing 
materials of different conductivity. The resulting models will then exhibit to some extent the behavior that the day temperatures are governed by the lowinertia material and the night temperatures by the high-inertia material. Thus some combination of these non-ideal properties could be the cause of the warm Martian nights. The infrared radiometers on Mariner 1971 (Chase et al. 1970), which will observe some areas at several local times, may allow the magnitude of these effects to be determined.

Comparatively small variations are expected in the density and specific heat of the Martian material which suggests that variations in thermal inertia may be directly interpreted as variations in conductivity. On Mars, surface densities are probably between 1.3 and $2.0 \mathrm{gm} \mathrm{cm}^{-3}$, a range that includes both lunar and terrestrial soils (Costes et al. 1970; Fryxell et al. 1970). The specific heat of many geologic materials, including silicates and carbonates, is near 0.19 at $300^{\circ} \mathrm{K}$ (Kieffer and Kamb 1971). The corresponding value at the average temperatures observed by Mariners 6 and 7 is 0.16 . Thus, for a density of 1.5 the representative value of $\rho c$ is 0.24 . The mean thermal inertia of 0.006 observed in the daytime, then, with a nominal correction of $-10 \%$ to account for atmospheric boundary-layer conduction (see Appendix), corresponds to a conductivity of $1 \times 10^{-4}$. Similarly, the greatest thermal inertia inferred, 0.010 , corresponds to a conductivity of $3 \times 10^{-4}$.

The comparatively extensive literature on the thermal properties of rock powders in a vacuum is largely inapplicable to Mars, where the conductivity of the atmosphere alone, approximately $2.2 \times 10^{-5}$ (pure $\mathrm{CO}_{2}$ at $200^{\circ} \mathrm{K}$ ), exceeds the conductivity of the lunar surface. The lowest conductivity observed for rigid geologic materials at Martian pressure is $K=3 \pm 1 \times 10^{-4}$ for a pumice of density 0.63 (Wechsler and Glaser 1965). Thus the thermal measurements require that the Martian surface be particulate, in accord with earlier thermal observations and interpretations of some visual cloud features.

In the range inferred for Mars, thermal conductivity is very dependent on particle size, relatively independent of porosity and, within the class of probable surface materials, independent of composition. Since the conductivity of solid rocks is greater than that of the Martian surface, any likely particulate material is compatible with the thermal observations if the particle size is chosen appropriately. The granularity of the surface can be estimated if it is assumed that the conductivity-particle size relation observed for silicate powders at $6 \mathrm{mbar}$ pressure (Wechsler and Glaser 1965, Fig. 4) is appropriate for the Martian surface. The conductivities derived above correspond to mean particle diameters of about $200 \mu$ and $1 \mathrm{~mm}$ for inertias of 0.006 and 0.010 , respectively. In this context it should be noted that the conductivity measured for basalt powder with grain sizes from 37 to $62 \mu$ (Fountain and West 1970) is considerably less than that inferred for the Martian surface. This interpretation apparently contradicts the suggestion, based on polarimetry, that the mean diameter of particles in the light areas is $50 \mu$ (Pollack and Sagan 1969); a thin surface dusting of small particles is, however, not excluded by the thermal data.

The atmospheric circulation calculations of Leovy and Mintz (1969) utilize an albedo range of 0.15 to 0.25 and a thermal inertia of 0.002 . The present data indicate that both these values are too small and that they will yield larger diurnal temperature variations than are representative for Mars. The wind velocities they calculate may correspondingly be somewhat too high.

\section{Relations of the Temperature Field with the Visual Images}

In order to exhibit possible correlations between the temperature field and visual features of the planet, the temperature $T_{10}$ and the differences $\Delta T=T_{20}-T_{10}$ have been plotted on the maps of Plates II-IV, which incorporate results of the imaging experiments of Mariners 6 and 7 (Leighton et al. 1969a, 1969b). Temperature variations between areas identified as dark or light in classical maps are readily recognized; an example is the $7^{\circ} \mathrm{K}$ difference measured with Mariner 6 between Thymiamata and Meridiani Sinus (Plate II). Mariner 7 data (Plate IV) also show a steep temperature gradient across the boundary between Hellespontus and Hellas, besides a number of fluctuations over Mare Tyrrhenum. The pronounced fluctuations on the dark part of the swath of Mariner 6 shown in Plate III may also largely reflect variations in the bolometric albedo around Syrtis Major, although here variations in the thermal inertia may also be present.

The most obvious characteristic of the Martian surface, besides the albedo differences between dark and light areas, are the craters. It is thus natural to seek temperature fluctuations which are clearly associated with craters. The only distinct correlation of this kind occurs near the large crater at longitude $19^{\circ} \mathrm{E}$ in Deucalionis Regio (Plate III and Fig. 3), which was viewed by the Mariner 6 radiometer slightly off center. Although the temperature of neighboring areas is changing steeply at this position because of local time, an increase of $0.6^{\circ} \mathrm{K}$ maximum amplitude, extending for about $85 \mathrm{~km}$ on the surface, can be seen. The observed temperature increase is a lower limit to the actual increase because of the smoothing effect of the extended field of view (Sec. I); the actual increase must be on the order of $1^{\circ} \mathrm{K}$. Neither the TV imagery of the area nor the topography inferred by $\mathrm{CO}_{2}$ absorption (Herr et al. 1970) gives clues as to whether this anomaly reflects changes in albedo and/or topography. 
A major objective of the radiometer experiment was to utilize the high spatial resolution available to find thermal anomalies present on the Martian surface. Although only approximately $2 \%$ of the Martian surface was observed, several thermal inhomogeneities on a spatial scale at the limit of the resolution of the radiometers have been detected; of special interest is the temperature increase observed by Mariner 6 around longitude $43^{\circ} \mathrm{W}$ (Plate I), because this region has been recognized in TV images as chaotic terrain (Sharp et al. 1971a). The maximum temperature was apparently not coincident with the darkest area of the TV image shown in Fig. 1 of Sharp et al. (1971a). Thus it is not clear that albedo variations alone account for this feature.

Among other temperature changes, the sharp decrease in $T_{10}$ which takes place at the southern boundary of the light region Aeria (Plate III, longitude $42^{\circ} \mathrm{E}$ ) is perhaps the most pronounced, although no sharp fluctuation in the albedo is known here. The second swath of Mariner 6 shown in Plate II also has several sharp changes which are only partially resolved by the radiometers. The apparent anticorrelation between $T_{10}$ and $\Delta T$ evidenced at these inhomogeneities is almost certainly an artifact of the broader angular response of the $18-25-\mu$ channel compared to the $8-12-\mu$ channel.

\section{The Temperature of the Polar Cap}

A major objective of the radiometer experiment was to measure the temperature of the condensable substance forming the southern polar cap of Mars. Since the temperature of the cap was suspected to be low, it was anticipated that only the $18-25-\mu$ channel would give useful data; in fact, the temperatures measured were sufficiently low so that this was the case.

Because the energy emitted by the cap is small compared with that prevailing in neighboring regions which are visually observed to be not covered with frost deposits, the effects of the extended fields of view and of possible thermal offsets in the radiometer are more significant than for the observations of other parts of the planet. The Mariner 7 TV pictures (Sharp et al. 1971b), show that the cap starts to become visible at a latitude of about $-59^{\circ}$ while south of $-62^{\circ}$ the coverage of the terrain by frost is nearly continuous. In order to perform the deconvolution of the field of view, a model surface temperature distribution which allowed for the presence of the polar cap itself was used. In the model adopted after some iterations, a temperature of $148^{\circ} \mathrm{K}$, the minimum uncorrected temperature observed on the cap, was assumed to hold for the cap proper. In the intermediate-latitude range between $-59^{\circ}$ and $-62^{\circ}$, the emitted energy was assumed to vary linearly between the values obtained from the unaltered thermal model at $-59^{\circ}$ and that corresponding to $148^{\circ}$ at $-62^{\circ}$ latitude. This thermal model, the

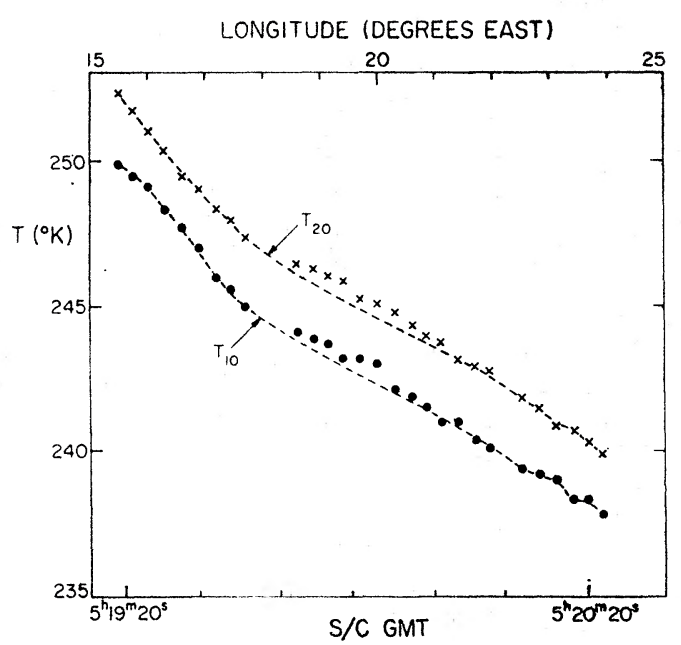

FIG. 3. The kinetic temperatures derived from Mariner 6 data near the crater at longitude $19^{\circ} \mathrm{E}$ in the Deucalionis Regio. The background (dashed lines) has been drawn to smoothly join data points between regions neighboring the crater.

raw data, and the data corrected as described in Sec. II are all shown in Fig. 4. It is seen that the over-all process is self-consistent, especially south of latitude $-73^{\circ}$. In fact, the corrections derived at this extreme section of the polar swath were found to be negligible in a variety of other calculations made with different thermal models and angular dependences of the response. Such was not the case for the region between $-62^{\circ}$ and $-72^{\circ}$, and therefore the temperature dip depicted there in Fig. 4 is not considered realistic.

When the instrument platform was slued to perform the swath which passed over the polar cap, the radiometer measurements of the internal calibration plate had a sudden offset from the values read previously. The offset remained constant during the entire polar pass and disappeared when the platform was reoriented for a more northerly view. Such an offset can be produced either by (i) a temporary gain change, (ii) energy reflected off the reference plate, or (iii) energy incident into the telescope during the view of "empty" space. Although it is impossible to uniquely differentiate between these possibilities, alternative (iii) seems the most plausible on the basis of postflight laboratory tests on the prototype model of the radiometer and the extreme geometry of the scan platform during the polar pass. This alternative was accepted and it effects a change of about $5^{\circ} \mathrm{K}$ in the derived temperatures of the cap with respect to those which would follow if the energy emitted by the calibration plate had remained constant.

It is concluded that the brightness temperature of the completely frost covered polar cap is $148_{-2}{ }^{+50} \mathrm{~K}$. The $5^{\circ} \mathrm{K}$ uncertainty is based on the ambiguity mentioned above; if this is removed $2^{\circ} \mathrm{K}$ is a reasonable estimate of the error due to noise, gain uncertainties, field of view corrections, etc. 


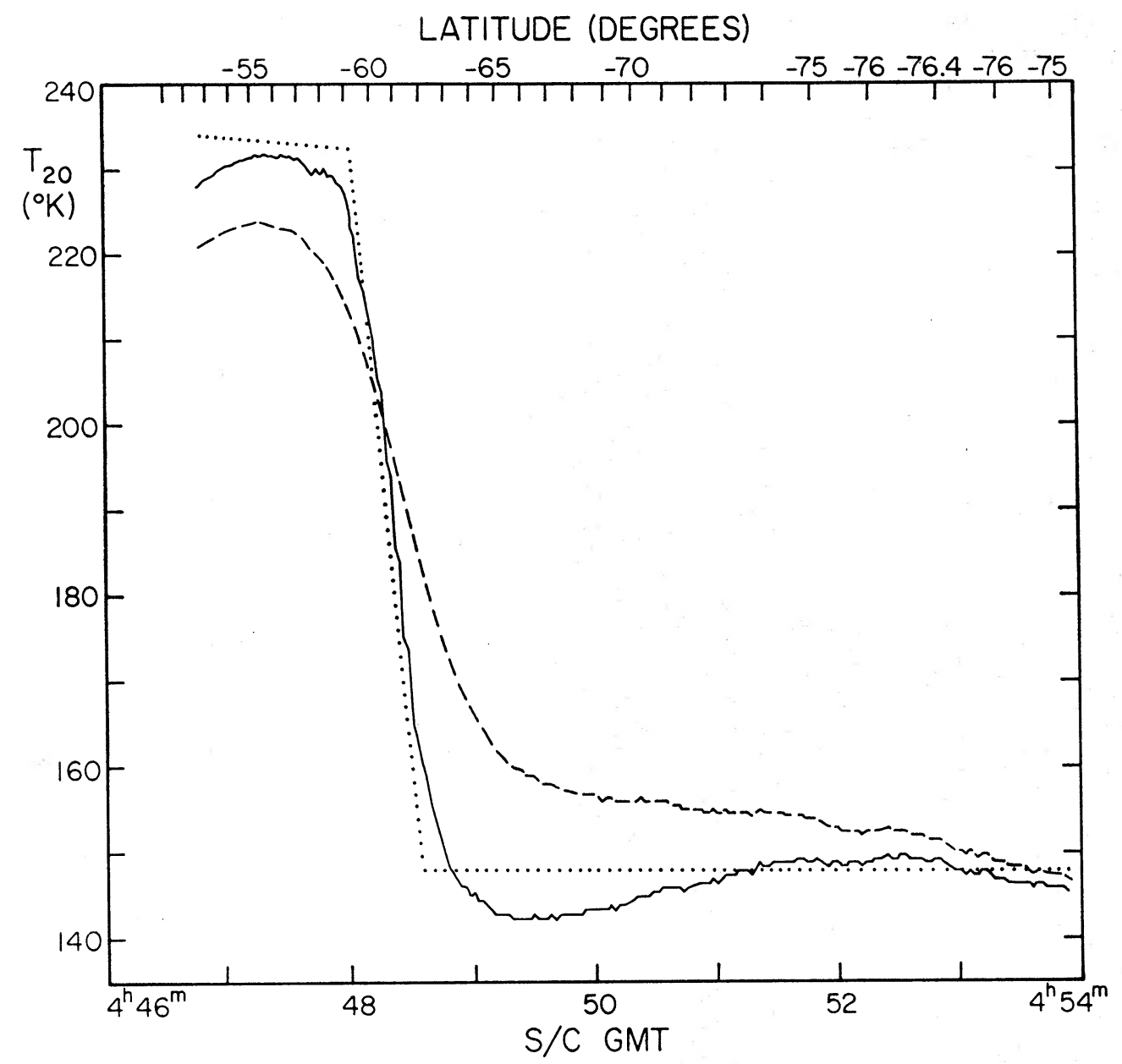

Fig. 4. The brightness temperatures observed near and on the southern polar cap: The dashed line represents the raw data while the solid line shows the data corrected for the effects of the extended field of view by assuming a model for the Martian surface temperatures represented by the dotted curve.
It is seen from Fig. 4 that the temperature of the frost covered area presents a striking change from that of the neighboring terrain. At the derived temperatures of $148^{\circ} \mathrm{K}$, the vapor pressure of $\mathrm{CO}_{2}$ is $6.4_{-1.6}{ }^{+6.4} \mathrm{mbar}$, a value close to the mean pressure derived from refractivity measures by Kliore et al. (1969) during the occultations of Mariners 6 and 7. The upper limit (12.8 mbar) is many standard deviations above the observational limit of $\mathrm{CO}_{2}$ pressure at mid latitudes and thus supports the hypothesis that no temporary gain changes occurred. The agreement between the vapor pressure of $\mathrm{CO}_{2}$ at $148^{\circ} \mathrm{K}$ and the mean atmospheric pressure at the surface is interpreted as strong evidence that $\mathrm{CO}_{2}$ is the predominant constituent of the Martian polar caps. In contrast, the vapor pressure of water ice at this temperature is about $4 \times 10^{-8}$ mbar. Herr and Pimentel (1969) have arrived at a similar conclusion on the basis of Mariner 7 infrared spectrometer observations. The implications of the hypothesis of $\mathrm{CO}_{2}$ frost were discussed by Leighton and Murray (1966) in general, and further by Sharp et al. $(1971 \mathrm{~b})$ in connection with the visual appearance of the polar cap.

\section{CONCLUSIONS}

The main results of the Mariner 6 and 7 radiometer experiments can be briefly summarized as follows:

(i) The maximum brightness temperature at $10 \mu$ measured on a dark area (Meridiani Sinus) agrees well with the value obtained by ground-based observations.

(ii) Temperatures of localized areas are largely correlated with the classical light and dark features in agreement with expectations based on the predicted range of bolometric albedos.

(iii) Local thermal inhomogeneities, both temperature increases and decreases, have been observed with spatial extent at the resolution limits of the radiometers.

(iv) On the illuminated hemisphere, the dependence of the surface temperature on local time indicates a mean thermal inertia $I=0.006$ for bolometric albedos in the range 0.20 to 0.40 . The highest albedo, 0.40 , is required for Hellas, independently of the assumed thermal inertia. The rate of change of temperature over this area implies $I=0.010$. The Deucalionis Regio area, observed by Mariner 6, requires the high albedo of 0.36 . 
(v) The nighttime temperatures are systematically higher than those of the homogeneous models compatible with daytime measurements. The darkest area observed by Mariner 6, Syrtis Major, requires a thermal inertia $I=0.009$ for an albedo of 0.15 . The region of Mare Tyrrhenum, observed by Mariner 7 at night, requires at least an equally high inertia. Although these observations offer circumstantial evidence for higher than average values of the inertia of the dark areas, the possibility of an intrinsic inconsistency between daytime and nighttime measurements has not been ruled out. The extremes of the inferred inertia imply that thermal properties of the Martian surface are determined by particles in the range 0.2 to $1 \mathrm{~mm}$ in diameter.

(vi) The temperature measured by Mariner 7 over the frost covered area of the south polar cap is $148^{\circ} \mathrm{K}$ and provides evidence that the major constituent is frozen $\mathrm{CO}_{2}$.

\section{ACKNOWLEDGMENTS}

The late $H$. Hatzenbeler was instrumental in the construction, testing, and calibration of the radiometers. We also thank D. Schofield and the members of the Mariner 1969 project for their help which made this experiment possible, J. Bennett and S. Law for their help in the reduction of the data, C. Macdonnell and M. Katz for help in preparation of the paper, and Dr. P. Gierasch for valuable discussions of the thermal interactions between the surface and atmosphere of Mars.

\section{APPENDIX: THE THERMAL MODEL}

The thermal model utilized an explicit solution to a finite difference scheme with exponentially increasing layer thicknesses scaled to the diurnal skin depth. The accuracy of the integrations was checked by independent calculations of homogeneous models utilizing the Laplace transform method of Jaeger (1953), with agreement to within $0.5^{\circ}$. Seasonal effects were not included.

For comparison with the radiometric data, a homogeneous model with temperature-independent physical properties was assumed. These assumptions were checked by relaxing them for a nominal Martian model as discussed below. Temperature effects on the thermal conductivity of particulate basalt under Martian conditions have been measured to be less than $10 \%$ over the temperature range of interest here (Fountain and West 1970). Calculation of the radiative contribution to thermal conductivity based on measurements by Watson (1964) indicate that this term, which has a $T^{3}$ dependence, will be only about $1 \%$ of the total conductivity. Hence temperature-independent conductivity is appropriate for Martian conditions when condensation processes are neglected and, therefore, was used for the thermal model.
Possible effects of variations of physical properties with depth were studied for a nominal Martian model by increasing the diffusivity $(K / \rho c)$ by a factor of 2 below 1 and $2 \mathrm{~cm}(0.27$ and 0.65 skin depths). These two-layer models differed from the homogeneous models by up to 6.8 and $4.2^{\circ} \mathrm{K}$, respectively. The main effect of increased diffusivity with depth is to raise temperatures through the night. For the range of local times observed by Mariner 1969, these models differed from the nearest fitting homogeneous model by at most $3^{\circ}$ and $2^{\circ}$, respectively. Measurements of conductivity and density under appropriate conditions (Wechsler and Glaser 1965; Fountain and West 1970) suggest that such variations of diffusivity are of as great a magnitude as might be expected for a dry particulate soil. The temperature changes due to such variations in the diffusivity with depth are smaller than those resulting from uncertainty of the albedo. The additional complexity of analysis involved in introducing two additional free parameters is probably not warranted in the absence of better albedo data. Thus only homogeneous models have been used for comparison with the observations.

The specific heat of geologic materials is very nearly directly proportional to temperature in the temperature range reported here (Winter and Saari 1969; Kieffer and Kamb 1971). Thermal models with this dependence were calculated, with results that differed by less than $1^{\circ} \mathrm{K}$ at all times from models with temperatureindependent specific heat.

The Martian atmosphere interacts with the surface thermal balance by both downward thermal emission and turbulent heat conduction across the boundary layer. Unpublished calculations by Gierasch (private communication) of radiative transfer in the atmosphere indicate that back radiation is small and nearly constant. Accordingly, a constant atmospheric back radiation equal to 0.01 of the local noon solar flux has been explicitly included in the thermal model.

The magnitude of turbulent heat transfer across the boundary layer is uncertain by about a factor of 2 (Gierasch and Goody 1968) and was not included in the thermal model. The effect of this process can be estimated by comparison of the surface temperatures attained in atmospheric models where the boundary layer parameters are varied. The change of the amplitude of the surface-temperature diurnal variation with the thermal inertia is $6.5 \times 10^{-3}$ for the mean Martian model. The theoretical turbulent heat transfer decreases the diurnal temperature amplitude $4.5^{\circ}$ (Gierasch and Goody 1968) and thus the expected effect of turbulent heat conduction is an apparent increase of the thermal inertia by about $10 \%$.

\section{REFERENCES}

Buhl, D., Welch, W. J., and Rea, D. G. 1968, J. Geophys. Res. 73, 5281.

Chase, S. C., Jr. 1969, Appl. Opt. 8, 639. 


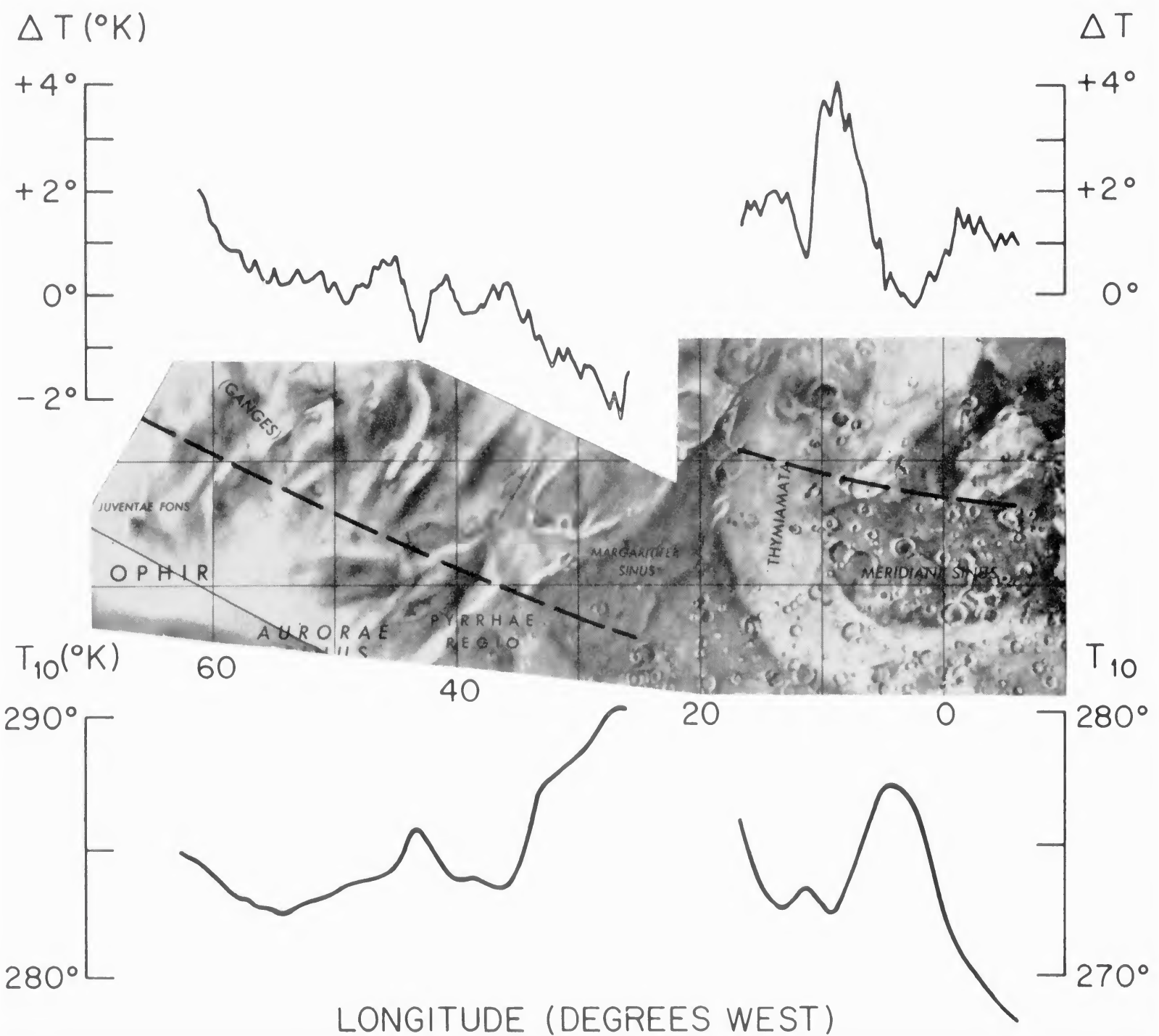

Plate II (Neugebauer et al., p. 719). The temperatures $T_{10}$ and differences $\Delta T=T_{20}-T_{10}$ measured along two swaths of Mariner 6 are shown in relation to the U. S. Army Topographic Command 1:25000 000 scale chart of Mars. The trace of the radiometers has been drawn from trajectory data. It may deviate in the most westerly swath from the trace of the TV camera-B shown by Sharp et al. (1971a, Fig. 2) by as much as 2 deg. For clarity, planetocentric latitudes have not been labeled; the latitudes of the map extend from $10^{\circ} \mathrm{N}$ to $20^{\circ} \mathrm{S}$ 


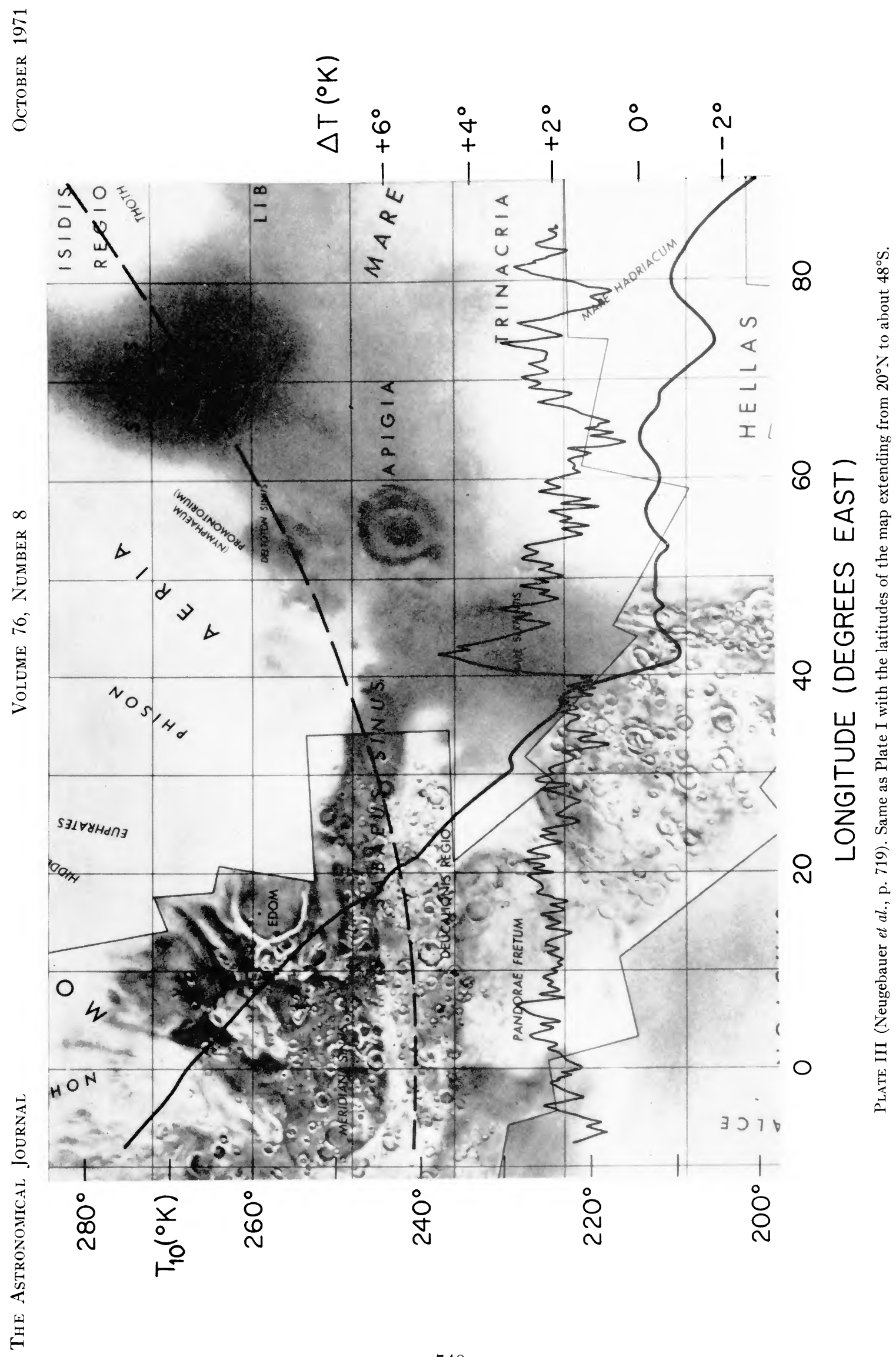




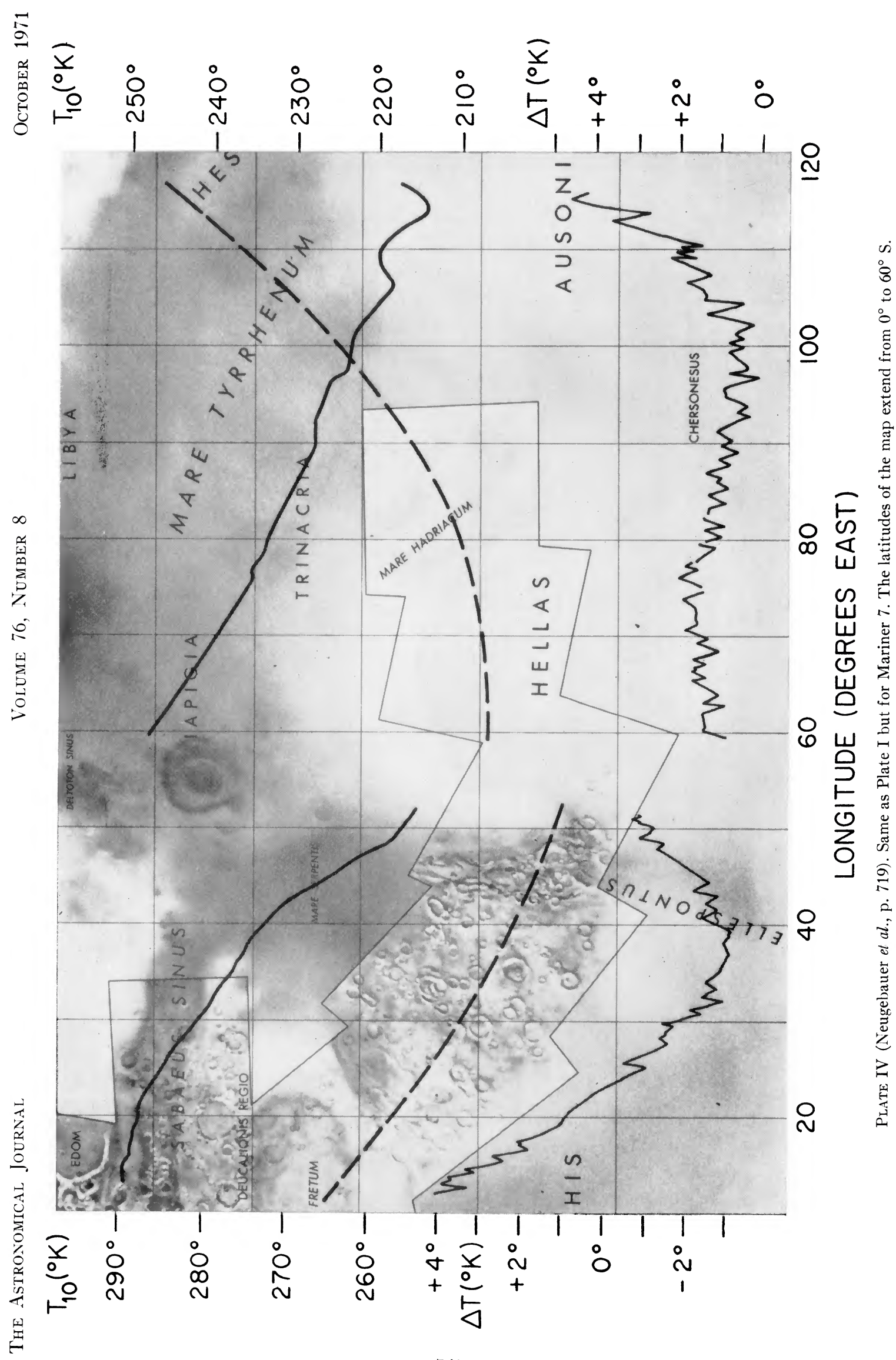

